\title{
THE MULTICOLOR FLUORESCENCE IN SITU HYBRIDIZATION (mFISH) HOMEPAGE
}

\author{
Liehr T*
}

*Corresponding Author: Dr. Thomas Liehr, Institut für Humangenetik und Anthropologie, Kollegiengasse 10, D-07743 Jena, Germany; Tel.: +49-3641-935533; Fax: +49-3641-935582; E-mail: i8lith@mti.uni-jena.de

\begin{abstract}
Multicolor fluorescence in situ hybridization (mFISH) assays are essential for a precise description of chromosomal rearrangements. Routine application of such techniques on human chromosomes started in 1996 with the simultaneous use of all 24 human whole chromosome painting probes in multiplex-FISH (M-FISH) and spectral karyotyping (SKY), even though the principle of mFISH was reported in 1989. Numerous approaches for chromosomal differentiation based on mFISH assays have been established, predominantly, to characterize marker chromosomes, but also in evolutionary biology, nuclear architecture, zoology and botany. The mFISH Homepage, which reviews all available literature at http://www.med. uni-jena.de/fish/mFISH/ mFISHlit.htm will be introduced here.
\end{abstract}

Key words: Molecular cytogenetics; Multicolor fluorescence in situ hybridization (mFISH); Metaphase FISH; Marker chromosomes; FISH-banding.

\section{INTRODUCTION}

The discipline of cytogenetics went through many stages of development, each providing more possibility for characterization of normal and structurally abnormal chromosomes [1]. Characteriza-

Institute of Human Genetics and Anthropology, Friedrich-Schiller-University Jena, Germany tion of such chromosomes in humans is of high clinical importance and is the requisite condition for further molecular investigation aimed at identification of disease-related genes [2,3] or positional cloning strategies [4]. Also, cytogenetic studies are significant for understanding the evolution of different animal species including Hominoids [5]. In the last 25-30 years, studies were accomplished by molecular cytogenetics, i.e., fluorescence in situ hybridization (FISH).

In 1969, Gall and Pardue [6], introduced in situ hybridization, which, for the first time, allowed nucleic acid sequences to be examined inside cells or on chromosomes. However, because radioactivity has well-known shortcomings [7], the radioactive probe for labeling was replaced by alternative labeling modalities or detection systems, e.g., biotin detected by binding to avidin coupled to a fluorochrome [8]. Pinkel and coworkers [9] described the FISH technique that could be applied to human chromosomes and very soon thereafter the first twocolor FISH study was published [10].

We have defined multicolor FISH (mFISH) as the simultaneous use of at least three different ligands or fluorochromes for the specific labeling of DNA (excluding the counter stain) [11]. According to this definition, the first successful mFISH experiments were those of Nederlof and coworkers [12] who visualized three differently-labeled nucleic acid sequences, simultaneously in blue [amino methyl coumarin acetic acid (AMCA)], in red [tetramethylrhodamine isothiocyanate (TRITC)] and in green [fluorescein isothiocyanate (FITC)]. 
Multicolor FISH assays have become indispensable for precise descriptions of chromosomal rearrangements, and studies including radiobiology, mutagenesis, inter-phase-architecture and chromosomal evolution. As the literature on this topic is huge, we created a regularly updated internet site in which all studies that apply mFISH are collected.

\section{CONTENT}

I have collected all the literature on mFISH and mFISH applications, and made the data available on the mFISH Homepage [11], which was created and has been available online since 2002. All literature that applies mFISH approaches according to our aforementioned definition is collected from publications available to the author and from PubMed (http:// www.ncbi.nlm.nih.gov/sites/ entrez) and is arranged according to the scheme shown in Figure 1. There are nine main categories: 1) Introduction gives basic information on the page; 2) Reviews lists papers that provide an overview on mFISH techniques and their pitfalls; 3) mFISH using wcps is subdivided into literature on M-FISH (multiplex-FISH)/ SKY (spectral karyotyping) applied in human Clinical Cytogenetics, Tumor Cytogenetics and Others; 4) Fish-banding summarizes all available approaches using human probes to achieve an mFISH-based chromosome banding; 5) CEP \& SC-probes reviews all papers on mFISH that use human centromeric and single copy probes; 6) Additional mFISH contains human mFISH probe sets not fitting any previous category; 7) $\mathbf{m F I S H}$ in animal and 8) $\mathbf{m F I S H}$ in plant are put together under these buttons. The corresponding subchapters can be seen in Figure 1. Under 9) mFISH probes available in cooperation and/or at cost price from the author of this article are listed.

The mFISH Homepage [11] can be used as a source of information in many ways. It can provide an overview into the importance of the mFISH field itself by taking note of the sheer volume of studies that apply multicolor molecular cytogenetics. It facilitates the search for mFISH reviews and basics. Also, it provides all relevant literature on already performed mFISH studies in clinical syndromes, special malignancies, mutagenesis, radiobiology, linkage/mapping, interphase-architecture and ZooFISH (Figure 2).

http://www. med.uni-jena.de/fish/mFISH/mFISHlit.htm

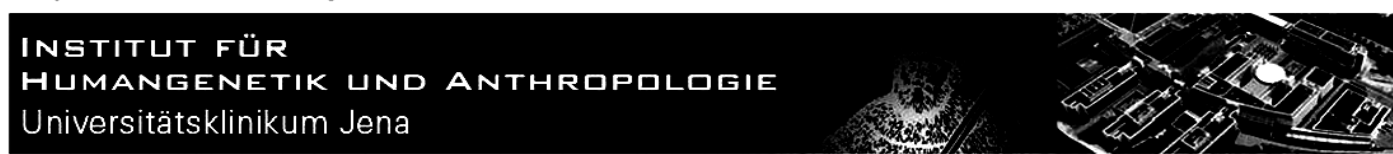

Page about multicolor fluorescence In situ hybridlzation (mFish) literature

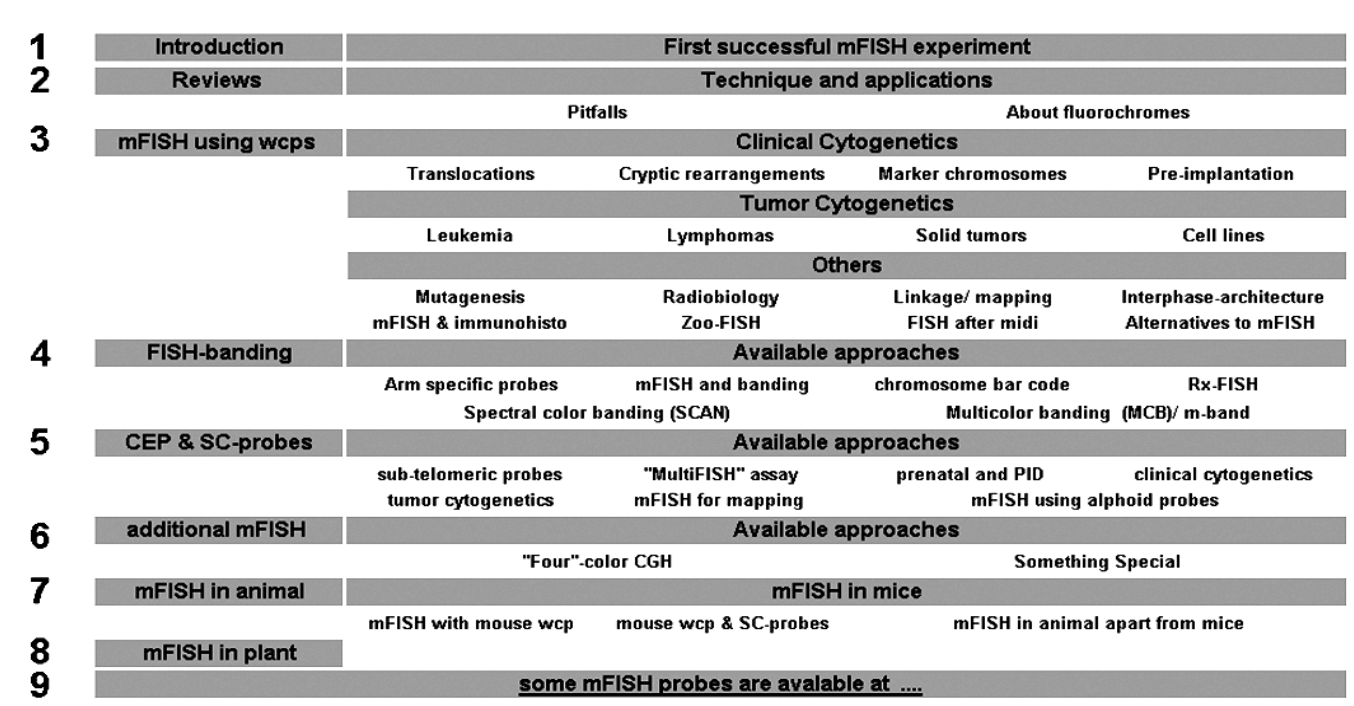

Figure 1. Interactive start page of the regularly updated mFISH Homepage. The figures 1-9 are not visible on the web page. 


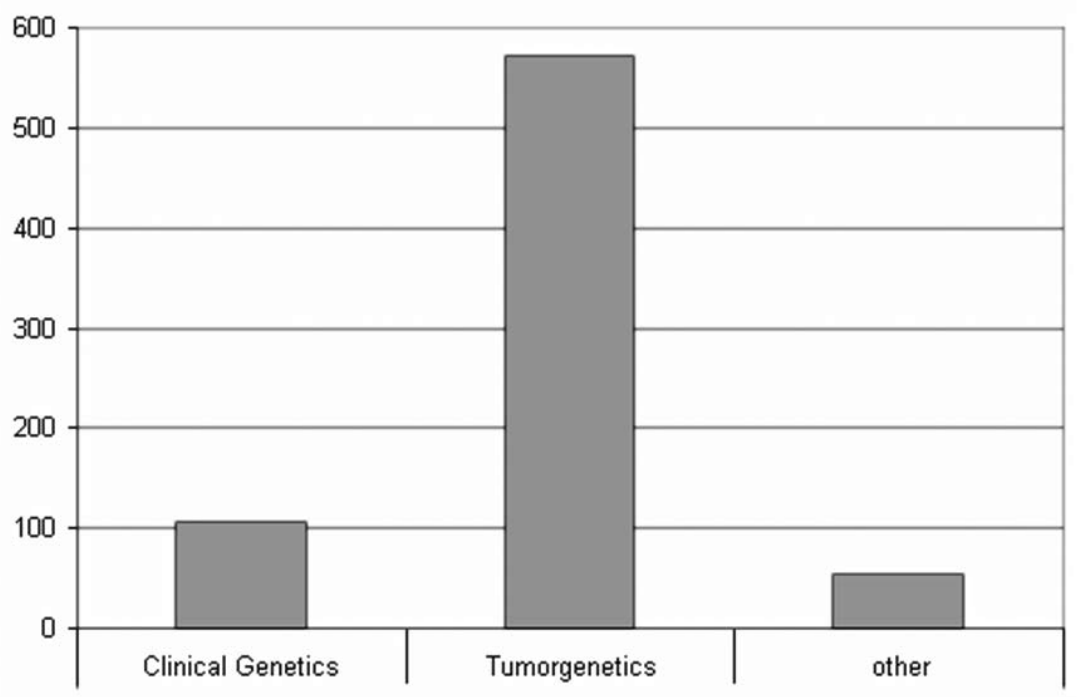

Figure 2. Number of published papers applying M-FISH/SKY in clinical genetics, tumor cytogenetics and other fields. Up to 04/2008 >700 publications used this mFISH approach, i.e., FISH using all 24 human whole chromosome painting libraries as probes, simultaneously.

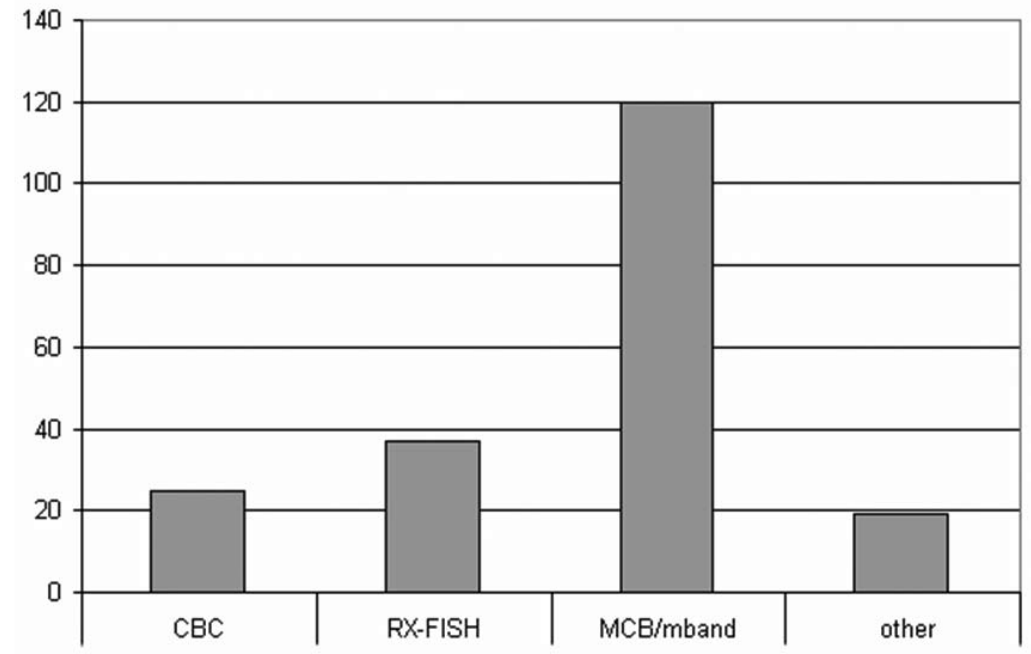

Figure 3. The FISH-banding approaches have been used in $>200$ research studies. The chromosome bar code (CBC), Rx-FISH and multicolor banding (MCB/ $\mathrm{m}$-band) are there most frequently applied.

It is always up-to-date on technical developments and new probe sets [11]. It provides information on mFISH combined with immunohistochemistry, microdissection after $\mathrm{mFISH}$, and alternatives to mFISH approaches. For FISH-banding [13], the following probe sets can be used: arm specific probes, M-FISH combined with banding, the chromosome bar code, Rx-FISH (cross-species color banding), spectral color banding (SCAN) and multicolor banding (MCB)/m-banding (Figure 3). Single copy probes and/or centromeric probes are applied in many different combinations and various applications; mFISH sets using sub-telomeric or microdeletion syndrome specific probes ("MultiFISH" assay) were created as well as for gene mapping, prenatal, preimplantation and clinical diagnostics 


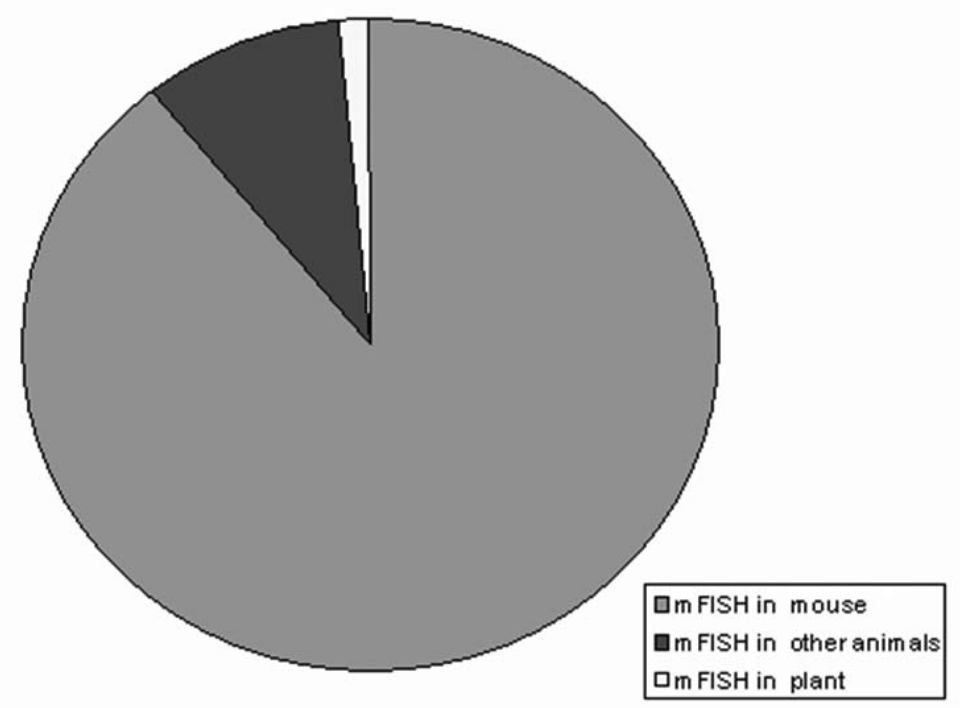

Figure 4. In animal and plants, mFISH is mainly applied in the mouse (Mus musculus).

or tumor cytogenetics. Furthermore, mFISH using alphoid probes, was developed for different applications, e.g., characterization of small supernumerary marker chromosomes [14].

While the aforementioned pages of the Homepage are mainly concentrated on human cytogenetics, mFISH applied in animals and plants are also included. Besides $\sim 60$ papers on $\mathrm{mFISH}$ using murine probes, also included are $\mathrm{mFISH}$ probe sets for Nasonia (Pteromalidae: Hymenoptera), chicken, rat, dog and horse (see FISH-banding) were reported in at least one publication, each. For plants only one $\mathrm{mFISH}$ application has been reported in Acricotopus (Figure 4). In summary, the mFISH Homepage is a valuable resource for all working in the diagnostic and research field of molecular cytogenetics.

\section{ACKNOWLEDGMENTS}

This study was supported in part by funds from the Stiftung Leukämie, Stefan-Morsch-Stiftung, DAAD 415-br-probal/po-D/07/09624 and D07/00070 and the DFG (LI820/11-1, LI820/171).

\section{REFERENCES}

1. Mitelman F. ISCN 1995: an international system for human cytogenetic nomenclature (1995): recommendations of the International Standing Committee of Human Cytogenetic Nomeclature, Memphis, TN, October 1994. Basel: Karger. 1995; $1-4$.

2. Rowley JD. A new consistent chromosomal abnormality in chronic myelogenous leukaemia identified by quinacrine fluorescence and Giemsa staining. Nature 1973; 243(5405): 290-293.

3. Becher R, Korn WM, Prescher G. Use of fluorescence in situ hybridization and comparative genomic hybridization in the cytogenetic analysis of testicular germ cell tumors and uveal melanomas. Cancer Genet Cytogenet 1997; 93(1): 22-28.

4. Collins FS. Positional cloning moves from perditional to traditional. Nat Genet 1995; 9(4): 347-350.

5. Yunis JJ, Prakash O. The origin of man: a chromosomal pictorial legacy. Science 1982; 215(4539): 1525-1530.

6. Gall G, Pardue ML. Formation and detection of RNA-DNA hybrid molecules in cytogenetical preparations. Proc Natl Acad Sci USA 1969; 63(2): 378-381.

7. Luke S, Shepelsky M. FISH: recent advances and diagnostic aspects. Cell Vis 1998; 5(1): 49-53.

8. Langer PR, Waldrop AA, Ward DC. Enzymatic synthesis of biotin-labeled polynucleotides: novel nucleic acid affinity probes. Proc Natl Acad Sci USA 1981; 78(11): 6633-6637. 
9. Pinkel D, Straume T, Gray JW. Cytogenetic analysis using quantitative, high sensitivity, fluorescence hybridization. Proc Natl Acad Sci USA 1986; 83(9): 2934-2938.

10. Hopman AH, Wiegant J, Raap AK, Landegent JE, van der Ploeg M, van Duijn P. Bi-color detection of two target DNAs by non-radioactive in situ hybridization. Histochemistry 1986; 85(1): 1-4.

11. Liehr T. mFISH Homepage at http://www. med. uni-jena.de/fish $/ \mathrm{mFISH} / \mathrm{mFISHlit.htm}$.

12. Nederlof PM, Robinson D, Abuknesha R, Wiegant J, Hopman AH, Tanke HJ, Raap AK.
Three-color fluorescence in situ hybridization for the simultaneous detection of multiple nucleic acid sequences. Cytometry 1989; 10(1): 20-27.

13. Liehr T, Starke H, Heller A, Kosyakova N, Mrasek K, Gross M, Karst C, Steinhaeuser U, Hunstig F, Fickelscher I, Kuechler A, Trifonov V, Romanenko SA, Weise A. Multicolor fluorescence in situ hybridization (FISH) applied to FISH-banding. Cytogenet Genome Res 2006; 114(3-4): 240-244.

14. Liehr T. sSMC homepage at http://www. markerchromosomes.ag.vu/ or http://www.med. uni-jena. de/fish/sSMC/00START.htm 
